\title{
Comprehensive analysis of blood cells and plasma identifies tissue-specific miRNAs as potential novel circulating biomarkers in cattle
}

\author{
Jason loannidis and F. Xavier Donadeu*
}

\begin{abstract}
Background: The potential of circulating miRNAs as biomarkers of tissue function, both in health and disease, has been extensively demonstrated in humans. In addition, circulating miRNA biomarkers offer significant potential towards improving the productivity of livestock species, however, such potential has been hampered by the absence of information on the nature and source of circulating miRNA populations in these species. In addition, many miRNAs originally proposed as robust biomarkers of a particular tissue or disease in humans have been later shown not to be tissue specific and thus to actually have limited biomarker utility. In this study, we comprehensively analysed miRNA profiles in plasma and cell fractions of blood from cattle with the aim to identify tissue-derived miRNAs which may be useful as biomarkers of tissue function in this important food animal species.

Results: Using small RNA sequencing, we identified 92 miRNAs with significantly higher expression in plasma compared to paired blood cell samples ( $n=4$ cows). Differences in miRNA levels between plasma and cell fractions were validated for eight out of 10 miRNAs using RT-qPCR ( $n=10$ cows). Among miRNAs found to be enriched in plasma, we confirmed miR-122 (liver), miR-133a (muscle) and miR-215 (intestine) to be tissueenriched, as reported for other species. Profiling of additional miRNAs across different tissues identified the human homologue, miR-802, as highly enriched specifically in liver.

Conclusions: These results provide novel information on the source of bovine circulating miRNAs and could significantly facilitate the identification of production-relevant tissue biomarkers in livestock. In particular, miR802, a circulating miRNA not previously identified in cattle, can reportedly regulate insulin sensitivity and lipid metabolism, and thus could potentially provide a specific biomarker of liver function, a key parameter in the context of post-partum negative energy balance in dairy cows.
\end{abstract}

Keywords: Circulating, microRNA, miRNA, Liver, Tissue-specific, Tissue-enriched, Biomarker, Cow, Small RNA sequencing, miR-802

* Correspondence: xavier.donadeu@roslin.ed.ac.uk

The Roslin Institute and R(D)SVS, University of Edinburgh, Easter Bush

Midlothian, UK

(c) The Author(s). 2018 Open Access This article is distributed under the terms of the Creative Commons Attribution 4.0 International License (http://creativecommons.org/licenses/by/4.0/), which permits unrestricted use, distribution, and reproduction in any medium, provided you give appropriate credit to the original author(s) and the source, provide a link to the Creative Commons license, and indicate if changes were made. The Creative Commons Public Domain Dedication waiver (http://creativecommons.org/publicdomain/zero/1.0/) applies to the data made available in this article, unless otherwise stated. 


\section{Background}

MicroRNAs (miRNAs) are short, non-coding RNA molecules which are primarily involved in the posttranscriptional fine-tuning of gene expression [1]. Since the discovery of lin-4 in Caenorhabditis elegans in 1993, thousands of miRNAs have been identified and characterised [2, 3]. It is now known that miRNAs are involved in the regulation of virtually all developmental processes, from the embryo to the adult [4-7], as well as in disease [8].

The discovery of cancer-associated miRNAs together with the detection of miRNAs in bio fluids [9] sparked a wave of research into the potential use of extracellular miRNAs as biomarkers of disease. A miRNA biomarker can be defined as a miRNA that is specifically produced or enriched in a given tissue, the circulating levels of which may reflect pathological or physiological changes in said tissue. In recent years, the value of circulating miRNAs as diagnostic biomarkers has been shown in relation to cancer (e.g. miR-21, miR-20, miR-221 [10, 11]), cardiovascular disease (miR-1, miR-133a [12]), liver disease (miR-122 [13]) and diabetes (miR-375 and miR-34 [14]), among many other human pathologies and physiological processes including pregnancy [15].

However, recent work has urged caution in interpreting the results of miRNA biomarker studies $[16,17]$. Specifically, a large number of miRNAs initially proposed as cancer biomarkers were later shown not to be tissue-specific, contrary to what would be expected from a robust tissue biomarker. Many of those miRNAs were found to be present in blood cells, which are a major contributor of miRNAs in circulation [16], and their circulating levels were shown to be affected by changes in blood cell numbers. In addition, a review of non-cancer, disease-related miRNA biomarkers found that the majority of proposed biomarkers were not tissue-specific and, moreover, were not expressed in the diseaserelevant tissues [18]. These reports highlight the need for further studies to identify the source of proposed miRNA biomarkers and determine their enrichment in the tissue(s) of interest.

The potential of miRNA biomarkers in foodproducing species has also been reported, although in a much smaller number of studies than in humans. The identification of biomarkers associated with specific production traits and disease conditions in cattle would be of significant agricultural importance. A handful of studies have already linked circulating miRNA profiles with pregnancy and the oestrous cycle $[19,20]$, infection $[21$, 22] and grazing [23] in cattle, as well as with bacterial disease and feed deprivation in other domestic species [24]. However, the source and tissue-specificity of circulating miRNA populations in these species have not been determined which, in view of the above, limits the practical significance of those results.
To address these limitations and facilitate the discovery in cattle of circulating bona-fide miRNA biomarkers, we sought to profile miRNA populations in different blood fractions in order to distinguish miRNAs expressed in blood cells from those expressed in other body tissues which could provide novel specific biomarkers. Using next-generation sequencing, we provide a list of potential tissue-enriched miRNAs in circulation including miR-802, a previously uncharacterised bovine liver-specific miRNA which may prove useful as a disease biomarker in high-producing cows.

\section{Methods}

\section{Sample collection}

Blood samples were collected in EDTA-coated tubes (Becton Dickinson, USA) from the jugular vein of six non-pregnant Holstein-Friesian cross cows, aged between 20 and 25 months and held at Langhill Farm (University of Edinburgh), with the relevant ethics approval (see Declarations). From each blood sample, a plasma fraction was obtained using a two-step centrifugation protocol [19] to separate blood cells [25] followed by filtration through $0.2 \mu \mathrm{m}$ syringe filters (Sartorius, Germany) to ensure the removal of any residual cellular debris. Subsequently, a cell fraction was obtained from a different volume $(100 \mu \mathrm{L})$ of the same blood sample by centrifugation at $1900 \mathrm{xg}$ for $10 \mathrm{~min}$ at $4{ }^{\circ} \mathrm{C}$. The resulting supernatant was discarded and the cell pellet (consisting of red blood cell and buffy coat fractions) was re-suspended in RNase-free water up to a volume of $250 \mu \mathrm{L}$. The blood cell solution was immediately used for RNA extraction as described below.

Bovine tissue samples including brain, heart, intestine, kidney (cortex and medulla combined), liver, lung, skeletal muscle, ovary, placenta (placentome and interplacentome combined), skin, spleen and uterus were collected from a local abattoir in ice-cold PBS. Upon arrival in the laboratory tissues were dissected, labelled, weighed and snap-frozen in dry ice. All samples were stored at $-80{ }^{\circ} \mathrm{C}$ until further use.

\section{RNA extraction}

All plasma, cell and tissue samples were extracted using TRIzol LS (Life Technologies, USA), as described [19], following the manufacturer's protocol. Blood cells $(250 \mu \mathrm{L})$ were homogenised using three volumes of TRIzol LS. Other tissues (each $50 \mathrm{mg}$ ) were thawed in $1 \mathrm{~mL}$ of TRIzol LS and homogenised with Lysing Matrix D (MP Biomedicals, UK) and a FastPrep FP120 Tissue Disruptor (Thermo Electron, USA). Before extraction, samples of plasma $(700 \mu \mathrm{L})$ and blood cells $(250 \mu \mathrm{L})$ were spiked with $3.5 \mu \mathrm{L}$ of exogenous cel-miR-39-3p (5.6 $\times$ $10^{8}$ copies per sample, Qiagen), and glycogen (40 $\mu$; Sigma-Aldrich, USA) was added in the presence of $1 / 10$ 
per volume of $5 \mathrm{M}$ ammonium acetate salt (Sigma-Aldrich), as recommended by the manufacturer, to facilitate RNA precipitation. RNA pellets from plasma, blood cells and other tissues were re-suspended, respectively, in 20, 10 and $40 \mu \mathrm{L}$ of RNase-free water (Qiagen) and used immediately or frozen at $-80{ }^{\circ} \mathrm{C}$ until use. RNA content and quality from cell and tissue samples were determined using the Nanodrop ND-1000 Spectrophotometer (Thermo Fisher Scientific, USA).

\section{Small RNA sequencing}

Small RNA libraries were prepared from four matched samples of blood plasma and cells using the TruSeq Small RNA Library Preparation Kit (Illumina, USA) and were submitted to 36-base single-end sequencing on the HiSeq 2000 Sequencing System (Illumina). Small RNA libraries were prepared using $5 \mu \mathrm{L}$ of RNA extract. Raw sequencing data, available on the GEO database (Accession GSE84871 [26]), were analysed using sRNAbench 1. $0[27,28]$. Briefly, the software was run in genome mode using default settings, with the bovine genome (bosTau4) and miRBase 21 as reference (accessed on 30 April 2015 $[29,30])$ in order to identify bovine (bta) miRNAs and human (hsa) miRNA homologues. Human homologues not previously described in bovine were identified by taking reads that aligned to the bovine genome but did not match with known bovine sequences in miRBase and aligning them to human sequences in miRBase. Throughout the manuscript, these miRNAs are indicated with the suffix hsa-. A single nucleotide mismatch was allowed when mapping reads to known miRNA sequences. Before mapping, reads without sequencing adaptor or with undetermined bases, and reads below 15 nucleotides in length (after adaptor removal) were removed and not used for subsequent analyses. For additional details about the integrated analysis steps in sRNAbench please refer to the software manual (http:// bioinfo2.ugr.es:8080/ceUGR/srnabench/).

Normalised reads (reads per million mapped, RPMM) obtained from sRNAbench were filtered before being passed to edgeR 3.10.2 for differential expression analysis in $\mathrm{R}$ language 3.2.1 [31, 32]. Prior to analysis, miRNAs which were detected with less than 25 RPMM in more than two samples of either plasma or cells were excluded in order to increase confidence in subsequent analysis and reduce technical noise. Differential expression analysis was performed using edgeR's paired mode on 212 miRNAs (Additional file 1) using GLMfit, as described in [33]. The statistical significance was set to false discovery rate (FDR) $<0.05$.

\section{Identification of potential novel biomarkers in cattle}

Potential novel tissue biomarkers were identified using the list of miRNAs which were enriched in plasma
(Additional file 1). MiRNAs that were either registered for bovine but not human, or that were human homologues not previously identified in cow were selected. To do this, Ensembl genome browser 85 (www.ensembl.org, [34]), NCBI (www.ncbi.nlm.nih.gov) and miRBase 21 (www.mirbase.org [35]) were used to identify miRNAs annotated in cow and human, and to determine their conservation between the two species. BLAST was also used (accessed via Ensembl at www.ensembl.org/Multi/ Tools/Blast?db=core, [36]) with default settings to identify the genomic location of selected miRNAs.

\section{RT-qPCR}

MiRNA levels were quantified in matched blood plasma and cell samples. For plasma samples, $2 \mu \mathrm{L}$ RNA were reverse-transcribed in a $10 \mu \mathrm{L}$ reaction using the miScript II RT Kit (Qiagen) in a Whatman-Biometra Thermocycler (Biometra, USA). For blood cell and other tissue samples, $500 \mathrm{ng}$ of RNA were used in each $10 \mu \mathrm{L}$ reaction. The cDNA template was diluted 40 -fold and added to $10 \mu \mathrm{L}$ qPCR reactions which were prepared in 96-well format using the miScript SYBR Green PCR Kit (Qiagen). Template amplification was carried out in an Agilent MX3000P qPCR system (Agilent Technologies, USA). Raw fluorescence data were collected using MxPro software (Agilent Technologies). The amplification efficiency ranged between $84.5 \%$ and $117.4 \%$, with $\mathrm{R}^{2}>0.86$. Expression levels were determined relative to a freshly-made standard curve and data were analysed using Microsoft Excel (Microsoft Corporation, USA). Expression levels were normalised to the mean expression of spiked-in cel-miR-39-3p for plasma and blood cells. For other tissues, expression data were normalised to RnU6-2.

Statistical analyses were carried out using GraphPad Prism 7 (GraphPad Software, USA). Differences in miRNA expression between blood plasma and cell samples were assessed using paired t-tests. In all cases, normality was tested using the Shapiro-Wilk normality test, outliers were tested with the ROUT test, and data were $\log _{2}(x+1)$ transformed to meet the tests' normality criteria. Statistical significance was set to $P<0.05$.

\section{Results and discussion}

\section{Small RNA sequencing of different blood fractions}

To determine the relative abundance of miRNAs in different blood compartments, we sequenced miRNAs in paired blood plasma and cell fractions from four cows. Analysis of read length distributions showed a peak at 20-23 nucleotides (characteristic of mature miRNAs) in blood cells and, although of lesser magnitude, in plasma (Fig. .1a). Unlike blood cells, plasma samples displayed an additional peak at 7-9 nucleotides. We attribute the higher proportion of short RNA fragments in plasma 

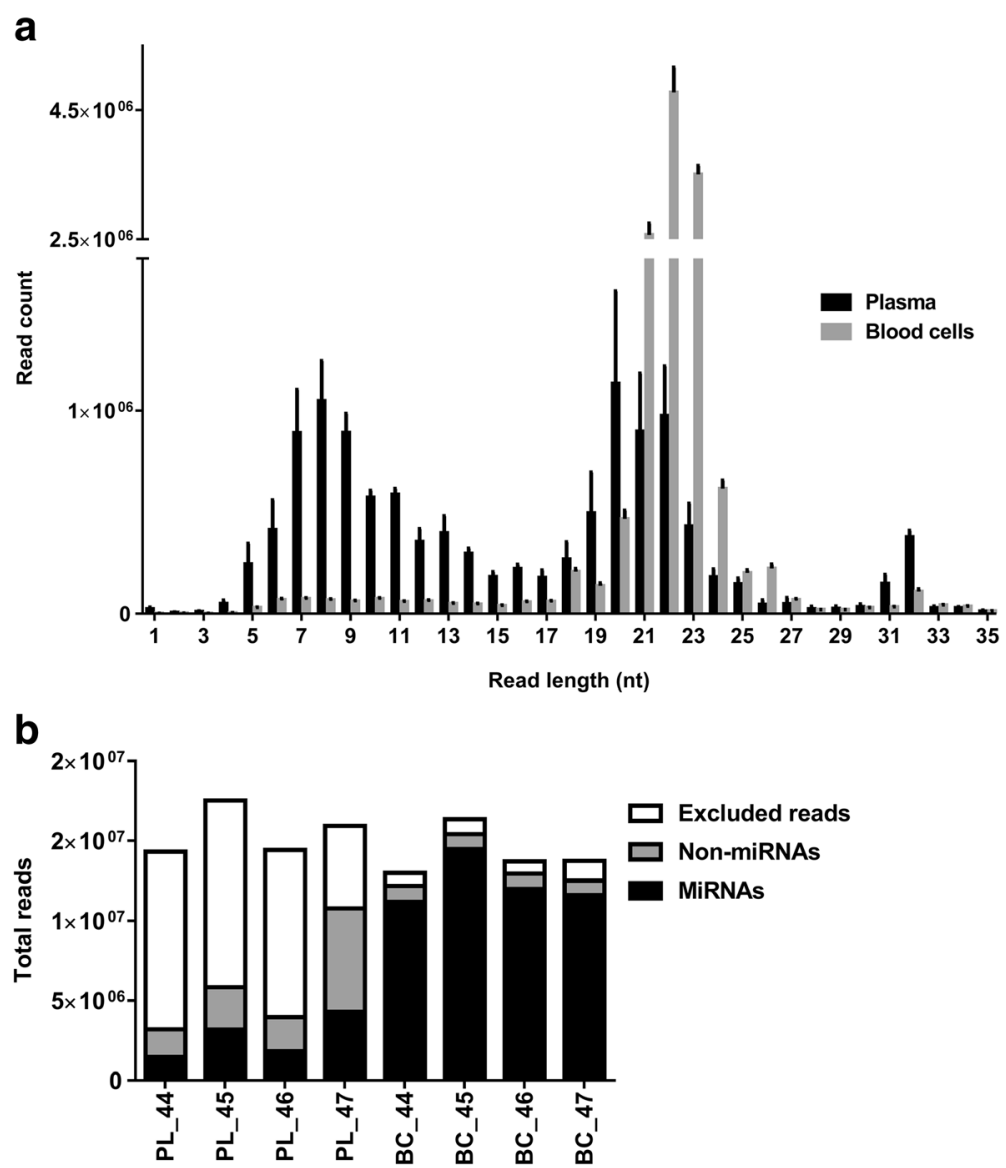

Fig.1 Results of small RNA sequencing analysis. a Length distribution (mean \pm SEM) of reads (before filtering out short reads) from paired plasma and cell samples of blood from four animals. b Bar plot showing the relative distribution of sequencing reads corresponding to miRNAs, non-miRNAs and excluded reads in individual blood plasma (PL) and cell (BC) samples

relative to cell samples to 1) the higher RNase content in plasma [9] resulting in higher levels of RNA degradation and 2) the relatively lower abundance of RNAs in plasma resulting in libraries with a higher proportion of adapter/adapter complexes. The difficulty associated with visualising low abundance cDNA bands on a gel during preparation of the plasma library likely resulted in many of the shorter sequences being included for sequencing.

Averaged for both plasma and cells, we obtained 14.9 million raw reads per sample (Table 1). After removing reads with low quality (reads without adapter, short reads and reads with multiple undetermined base calls), we mapped on average 5.9 million and 13.3 million reads to the bovine genome (see Methods) which correspond to $38.1 \%$ and $94.3 \%$ of the total reads for plasma and cells, respectively (Table 1, Fig. .1b). On average, plasma generated 2.7 million miRNA reads compared to 12.3 million reads for cells, the majority of which were, in both cases, bovine miRNAs (Table 1, Fig. .1b). After applying a detection threshold of 10 reads per sample, we
Table 1 Distribution of sequencing reads (RPMM) from paired blood plasma and cell samples ( $n=4$ cows)

\begin{tabular}{lll}
\hline & Plasma & Cells \\
\hline Raw reads & $15,553,150$ & $14,194,750$ \\
Reads without adapter (excluded) & 127,738 & 237,726 \\
Short reads (excluded) & $9,469,537$ & 672,891 \\
Low quality reads (excluded) & 20,587 & 25,532 \\
Mapped reads & $5,935,288$ & $13,258,602$ \\
Total miRNA reads & $2,688,243$ & $12,309,110$ \\
of which bovine & $2,683,651$ & $12,307,733$ \\
of which human & 4592 & 1377 \\
Detected miRNAs (> 10 reads) & 315 & 305 \\
of which bovine & 298 & 289 \\
of which human & 17 & 16 \\
\hline
\end{tabular}


Table 2 Most abundant miRNAs in paired blood plasma and cell samples ( $n=4$ cows) obtained by sequencing

\begin{tabular}{lllll}
\hline Plasma & & & Cells & \\
\cline { 5 - 5 } MiRNA & RPMM & & MiRNA & RPMM \\
\hline miR-22-3p & 126,167 & & miR-486 & 324,749 \\
miR-486 & 125,775 & & miR-451 & 187,081 \\
miR-192 & 101,853 & & miR-101 & 90,473 \\
miR-27b & 43,371 & & miR-92a & 44,722 \\
miR-142-5p & 39,727 & & miR-16b & 33,980 \\
miR-423-5p & 35,409 & & miR-26a & 32,849 \\
miR-191 & 32,448 & & miR-25 & 30,232 \\
miR-215 & 28,947 & & miR-142-5p & 20,925 \\
miR-21-5p & 25,484 & miR-191 & 19,656 \\
miR-10b & 25,277 & miR-181a & 15,753 \\
miR-92a & 23,608 & miR-186 & 14,137 \\
miR-103 & 22,481 & let-7f & 12,830 \\
miR-140 & 19,501 & miR-30e-5p & 11,955 \\
miR-148a & 19,108 & miR-144 & 9847 \\
miR-30e-5p & 19,058 & miR-93 & 9760 \\
\hline
\end{tabular}

detected up to 315 unique miRNAs in plasma samples and 305 in cell samples, of which only 17 and 16, respectively, were human homologues (Table 1).

Differentially expressed miRNAs in blood plasma and cells There was incomplete overlap between the lists of most abundant miRNAs in plasma and cells, with only 5 of the 15 most abundant miRNAs being common to the two lists, i.e. miR-486, miR-142-5p, miR-191, miR-92a and miR-30e-5p (Table 2).

Principal component analysis (PCA) of a total of 212 miRNAs that were detected with $\geq 25$ RPMM in more than two samples of either plasma or cells (see Methods, Additional file 1) produced well-separated plasma and cell populations, with a single component (PC1) accounting for $83.9 \%$ of the variation across samples (Fig. 2a). Moreover, 169 miRNAs were differentially expressed between plasma and cells (FDR $<0$. 05, Fig. 2b, Additional file 1), with as many as 131 miRNAs changing by more than two-fold. Of these, 92 miRNAs were enriched in plasma and 39 miRNAs were enriched in blood cells (Table 3, Fig. 3 and Additional file 1). Among miRNAs enriched in plasma were several known to be expressed exclusively or predominantly in specific tissues in humans including miR-122 (liver), miR-133a (muscle), miR-127 (adrenal gland), miR-141 (adrenal gland and reproductive system) and miR-182 (thymus) [37, 38]. Moreover, miRNAs enriched in the bovine blood cell fraction included many previously reported to be blood cellderived in humans, such as miR-144, miR-451, let-7f,
miR-26a, miR-15b, miR-20a, miR-16a, miR-16b and miR-486 [18, 25, 37, 39-41].

\section{Validation of sequencing results using RT-qPCR}

We used RT-qPCR to validate differences involving a total of 10 miRNAs (indicated in bold in Additional file 1) using samples from a total of six animals (including the four animals used for sequencing). Levels of one of the miRNAs selected for validation (miR429) could not be accurately quantified in most samples because of its low abundance. For the remaining nine miRNAs (Fig. 4a) we confirmed differences $(P<0.05)$ in the levels of all but miR-375 between plasma and cell samples (Fig. 4b). We then sought to validate the results for these eight miRNAs (miR-122, miR-215, miR-133a, miR-144, miR-451, and miR-6119-5p, miR-26a and let-7f) using samples from an independent group of animals (four HolsteinFriesian cross cows, aged between 24 and 48 months, in late pregnancy or post-partum) and we confirmed differences in plasma and cell levels of seven miRNAs (Additional file 2); levels of miR-133a were very low (Ct 34-37) in the original group of animals and were undetectable in this second group.

\section{MiRNA profiling across bovine tissues}

To identify novel tissue-specific miRNAs in cattle we investigated the distribution across 14 different body tissues of several miRNAs which expression we had found to be enriched in plasma (Additional file 1). We first profiled three miRNAs, miR-122, miR-133a and miR215 , known to be tissue-specific in humans [37, 38]. Accordingly, the three miRNAs (Fig. 5a) were expressed predominantly in liver (miR-122, 88-fold higher than in any other tissue), muscle/heart (miR-133, 254-fold higher) and intestine (miR-215, 150-fold higher).

We then sought to identify novel tissue biomarkers. First, from the list of miRNAs enriched in plasma (Additional file 1), we looked for miRNAs that were either registered for bovine but not human, or that were human homologues not previously identified in cow, as described in the Materials and Methods. These analyses revealed 10 miRNAs which either were present in bovine but not in human (miR-1388-5p, miR-1388-3p, miR2376, miR-2285 t, miR-3431 and miR-6529a, Fig. 5b) or were homologues of human miRNAs not identified previously in cow (miR-210-3p, miR-802, miR-4532 and miR-4792, Fig. 5c).

RT-qPCR analyses showed that the majority of these miRNAs were expressed in most of the tissues profiled, i.e., they were not enriched in any particular tissue and therefore do not provide potential biomarkers of tissue function (Fig. 5b-c). Surprisingly, miR-1388-3p, miR2376 and miR-6529 showed high expression in blood 

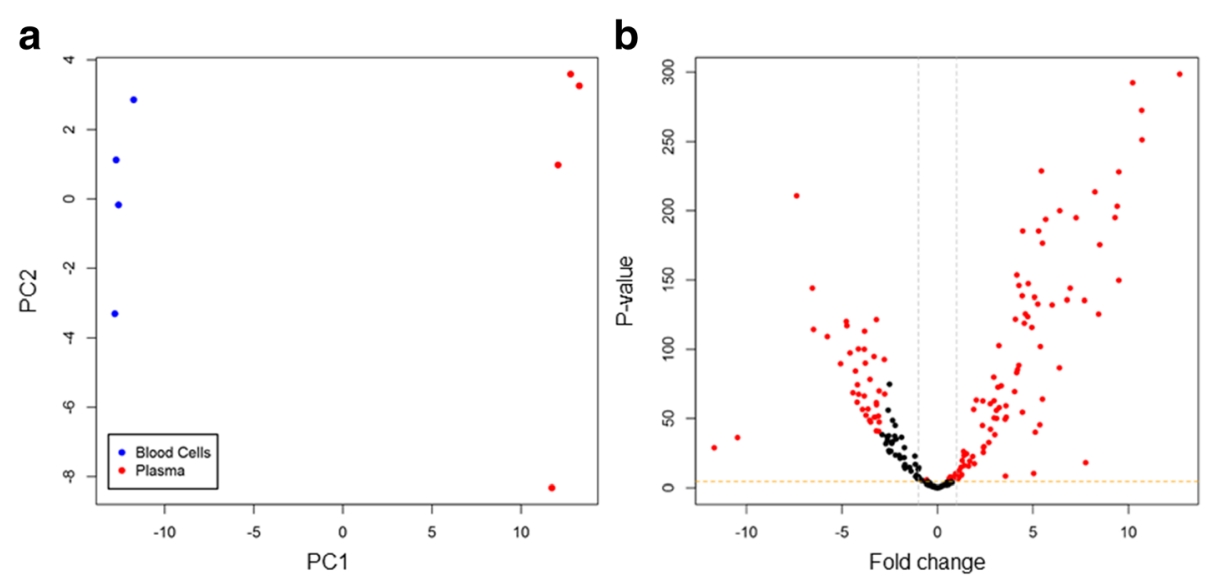

Fig. 2 Small-RNA sequencing results. a PCA plot showing the first two principle components using transformed normalised data. $\mathbf{b}$ Volcano plot of fold-change (miRNA levels in blood plasma relative to cells) versus statistical significance ( $P$-values), using transformed normalised data. MiRNAs with fold-change $>2$ and FDR $<0.05$ are highlighted in red. Grey dotted lines indicate two-fold difference thresholds. The orange dotted line indicates $P=0.05$. Paired blood plasma and cell samples from four animals were analysed

cells despite having been identified as plasma-enriched using sequencing. A possible explanation for this result is that, despite blood cells having the highest levels of these miRNAs, the combined contribution from all other tissues to plasma levels is still greater in comparison. Interestingly, in addition to blood cells, all three miRNAs were relatively abundant in spleen (Fig. 5b), which suggests an involvement in hematopoietic regulation. However, since no functional data is available for any of the three miRNAs in any species, further study will be needed to confirm this.

Among the remaining miRNAs, miR-3431 was distinctly high in lung followed by placenta and uterus.
This is a ruminant-specific miRNA which has been reported to be expressed in adipose and reproductive tissues of cattle [42, 43], although no information is available on its potential functions. Whether miR-3431 could provide a useful circulating biomarker of lung function in cows should be investigated in future studies.

Of note, the human homologue of miR-802 was distinctly enriched in liver, where its expression was 87-fold higher than the mean expression across all tissues, and 5.2-fold higher than in intestine, the tissue with the second highest mean expression levels (Fig. 5c). Using BLAT (UMD3.1, accessed 27/07/16) we mapped the mature miR-802 sequence to bovine chromosome 1

Table 3 The 15 most enriched miRNAs in blood plasma and cell samples ( $n=4$ cows) obtained by sequencing

\begin{tabular}{llll}
\hline Plasma-enriched miRNAs & Fold-change Plasma/Cells & Blood cell enriched miRNAs & Fold-change Cells/Plasma \\
\hline miR-429 & Expressed exclusively in plasma & miR-107 & 54.19 \\
miR-200a & 6503.71 & miR-6119-5p & 28.01 \\
miR-205 & 5220.54 & miR-185 & 27.94 \\
miR-215 & 2168.64 & miR-144 & 16.56 \\
miR-455-5p & 1707.35 & miR-451 & 10.31 \\
miR-100 & 1498.54 & miR-331-3p & 8.55 \\
miR-199a-5p & 1359.19 & miR-454 & 7.92 \\
miR-10b & 1339.56 & miR-339a & 7.61 \\
hsa-miR-802 & 1092.70 & let-7f & 7.16 \\
miR-199c & 1041.52 & miR-23b-3p & 6.53 \\
miR-214 & 931.10 & miR-26a & 6.08 \\
miR-141 & 699.78 & miR-98 & 5.72 \\
miR-455-3p & 635.12 & let-7a-5p & 5.60 \\
miR-122 & 493.42 & miR-101 & 5.39 \\
miR-126-5p & 412.95 & miR-197 & 5.36 \\
\hline
\end{tabular}



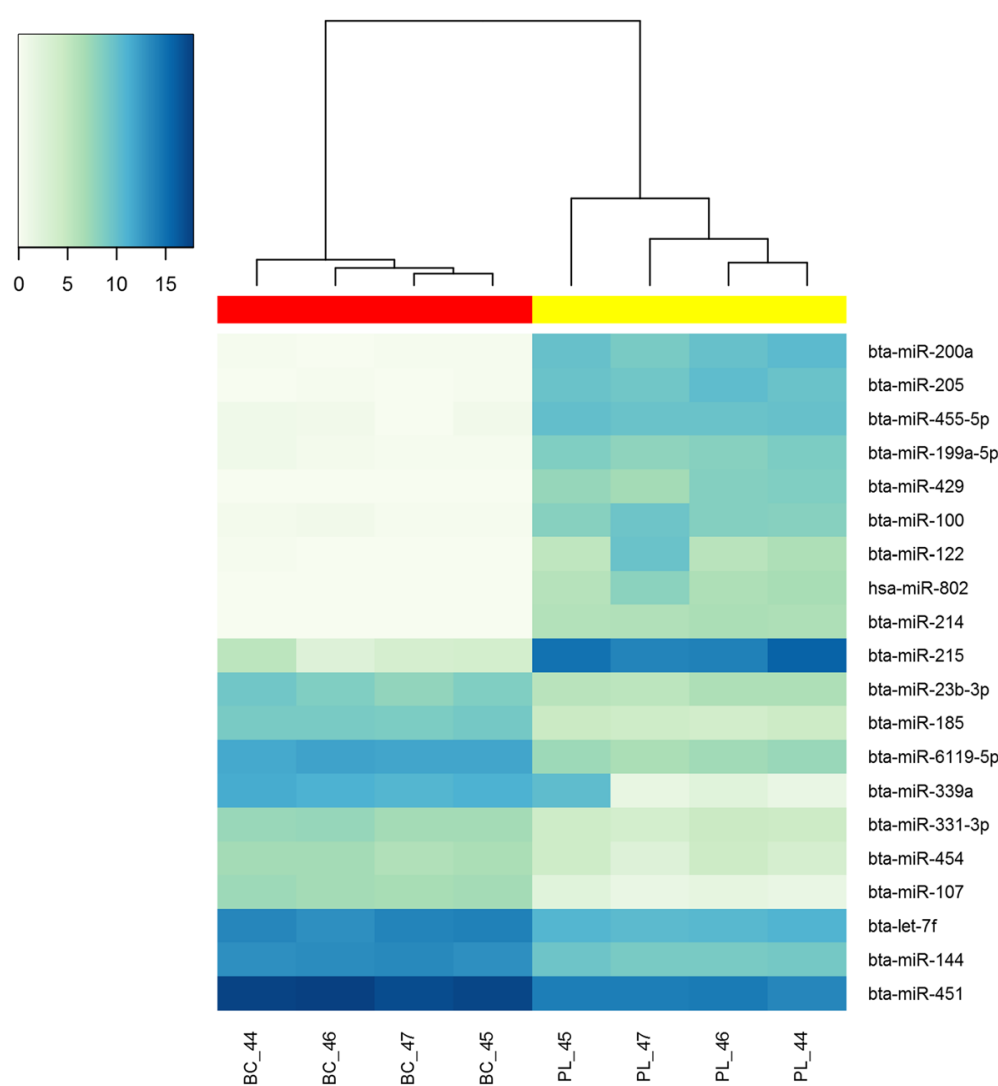

Fig. 3 Heat map of top miRNAs differentially expressed in paired samples of blood plasma (PL) and cells (BC). Each row in the heat map represents a miRNA and each column represents a sample. The colour scale illustrates the relative expression level of miRNAs. FDR $<0.05, n=4$ cows

(chr1: 149720302-149,720,392 [+]). Indeed, this location in the bovine genome has been annotated as a novel miRNA with a secondary structure that matches miR802. Although not previously reported in cow, data in humans and mice have shown miR-802 to be a key a regulator of liver function. Specifically, hepatic levels of miR-802 were elevated in obese individuals, contributing to insulin insensitivity, glucose intolerance and increased hepatic gluconeogenesis by downregulation of HNF1B [44]. Interestingly, serum miR-802 levels were reportedly increased in type 2 diabetes patients providing a potential circulating biomarker for this disease [45]. Another study identified miR-802 as a target of constitutive androstane receptor (CAR) signalling, which is involved in the regulation of xenobiotic detoxification, lipid homeostasis and energy metabolism [46]. Furthermore, circulating miR-802 has been proposed as a biomarker of drug-induced liver damage in rats, with an observed increase in miRNA levels in response to tissue damage being comparable to that of the liver-enriched miR-122 $[47,48]$. Overall, these results highlight the importance of miR-802 in the regulation of glucose and lipid metabolism as well as xenobiotic responses in the liver.
In dairy cattle, negative energy balance (NEB) often occurs during the post-partum period as a consequence of the high energy requirements for lactation. The liver has a central role in counteracting NEB. Liver dysfunction often ensues during the post-partum period, particularly in high-producing cows, in association with metabolic dysfunction and low fertility, constituting a major problem in modern dairy herds [49]. Changes in the expression of some miRNAs in liver have been shown in association with NEB in cattle [49]. In this context, future studies should investigate whether miR802, perhaps in combination with liver-enriched miR122, may provide a potential biomarker of negative energy balance in dairy cows.

\section{Conclusions}

Using next-generation sequencing, we compared for the first time miRNA levels in paired plasma and cell fractions of bovine blood and identified a total of 131 miRNAs that were differentially expressed (greater than two-fold) between the two fractions. Of those, 92 miRNAs were expressed at higher levels in plasma than in cells. Expression profiling of selected plasma- 


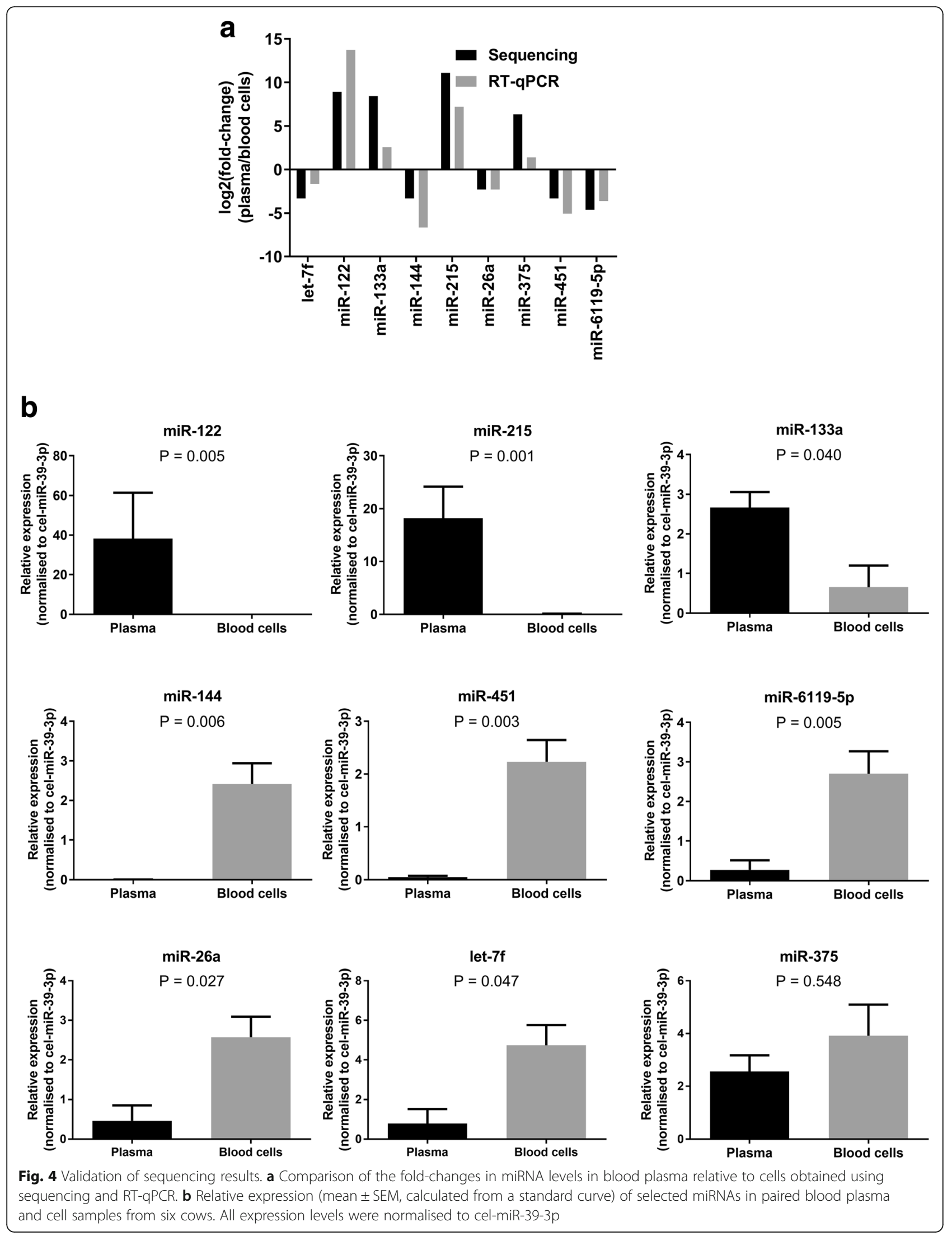




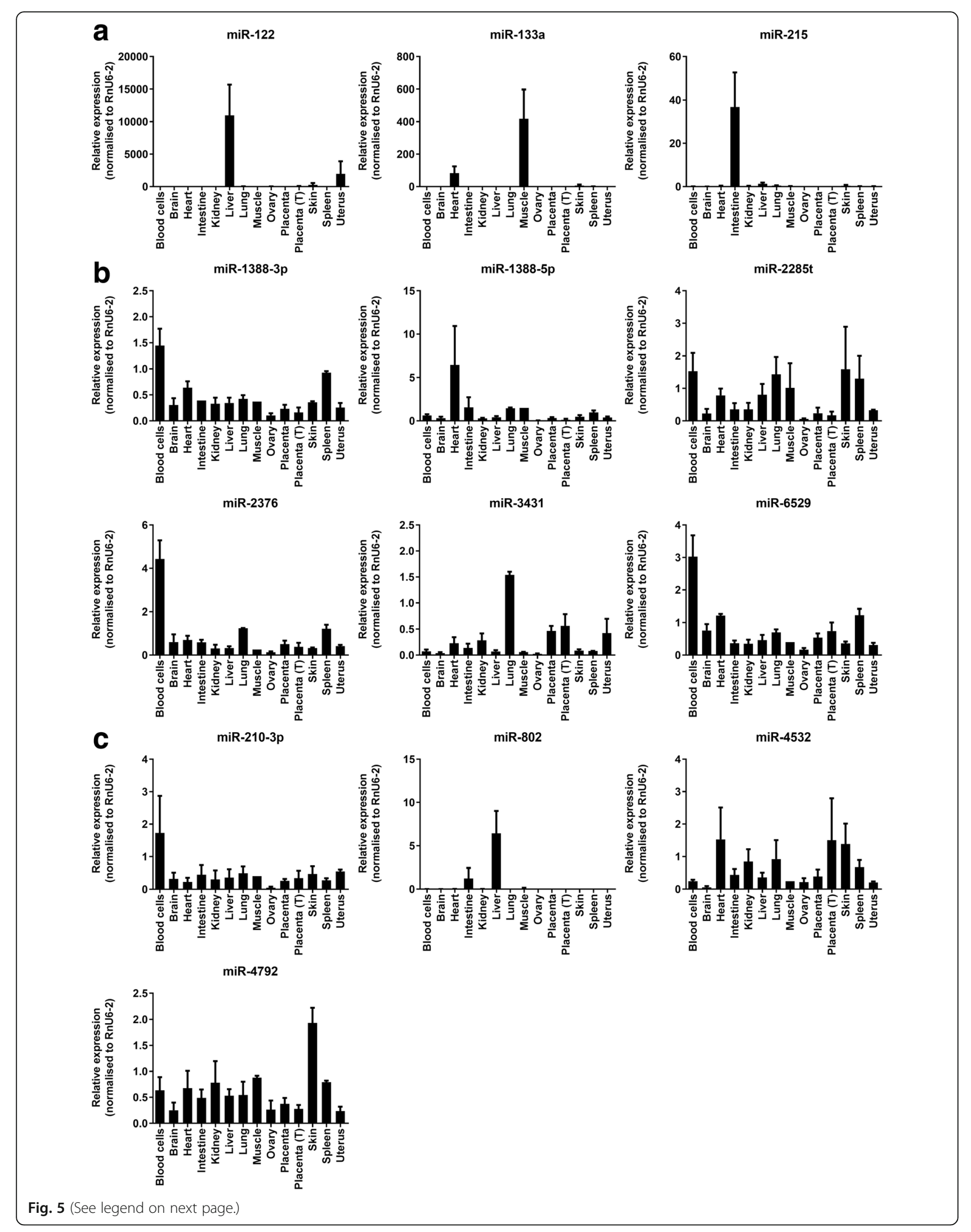


(See figure on previous page.)

Fig. 5 MiRNA profiling across bovine tissues. Relative expression (mean $\pm S E M$ ) of selected plasma-enriched miRNAs across 14 bovine tissues. a MiRNAs reported to be tissue-enriched in humans. b MiRNAs not registered in humans. c Human miRNA homologues not identified previously in bovine. RT-qPCR was run on 2-6 samples per tissue. Placenta = Day 70 of pregnancy; Placenta $(T)=$ Term. All expression levels were normalised to RNU6-2

enriched miRNAs across different bovine tissues revealed miR-802, a previously unidentified bovine miRNA, to be highly enriched in liver. The miRNA database generated in this study provides a useful resource for future investigation of miRNA biology in bovine and identification of additional potential biomarkers of tissue function in livestock.

\section{Additional files}

Additional file 1: Sequencing miRNA analysis. Normalised miRNA expression levels in bovine blood plasma and cell samples, and the results of differential expression analysis between the two sample types. (XLSX $47 \mathrm{~kb}$ )

Additional file 2: Validation of sequencing data. Validation of sequencing results for selected miRNAs using RT-qPCR on an independent group of four animals. (TIFF $1669 \mathrm{~kb}$ )

\section{Abbreviations}

miRNA: microRNA; PCA: Principal component analysis; qPCR: Quantitative polymerase chain reaction; RT: Reverse transcription

\section{Acknowledgements}

We thank Judith Risse, Helen Gunter and Mick Watson for assistance in the interpretation of sequencing data. We also thank Cheryl Ashworth for providing valuable feedback throughout the study and Helen Brown for helping with statistical analyses. Finally, we are very thankful to Tim King and Wilson Lee for advice in planning the animal study and carrying out the animal work, and to Bushra Mohammed and Sadanand Sontakke for assisting with the collection of tissue samples.

\section{Funding}

This study was funded by Zoetis, Inc. and the Biotechnology and Biological Sciences Research Council (BBSRC). The funding bodies did not participate in study design, data collection, analysis, interpretation or writing of the manuscript.

\section{Availability of data and materials}

The datasets generated and/or analysed during the current study are available in the GEO repository, Accession GSE84871, www.ncbi.nlm.nih.gov/ geo/query/acc.cgi?token=olgtysuirzqfpmr\&acc=GSE84871 [26].

\section{Authors' contributions}

$J$ participated in the design of the study, performed the experiments, analysed and interpreted data, and wrote the manuscript. FXD conceived the study and participated in study design, interpretation of data and writing the manuscript. Both authors read and approved the final manuscript.

\section{Ethics approval}

All animal procedures were carried out under the UK Home Office Animals (Scientific Procedures) Act 1986, license 60/4604, with approval by the Ethical Review Committee, University of Edinburgh.

\section{Competing interests}

This study was partially financed by Zoetis, Inc.

\section{Publisher's Note}

Springer Nature remains neutral with regard to jurisdictional claims in published maps and institutional affiliations.

Received: 19 April 2017 Accepted: 3 April 2018

Published online: 10 April 2018

\section{References}

1. Hammond SM. An overview of microRNAs. Adv Drug Deliv Rev. 2015; 87:3-14.

2. Lee RC, Feinbaum RL, Ambros $V$. The $C$. Elegans heterochronic gene lin-4 encodes small RNAs with antisense complementarity to lin-14. Cell. 1993;75:843-54

3. Casey MC, Kerin MJ, Brown JA, Sweeney KJ. Evolution of a research field-a micro (RNA) example. PeerJ. 2015;3:e829.

4. Motti D, Bixby JL, Lemmon VP. MicroRNAs and neuronal development. Semin Fetal Neonatal Med. 2012;17:347-52.

5. Finch ML, Marquardt JU, Yeoh GC, Callus BA. Regulation of microRNAs and their role in liver development, regeneration and disease. Int J Biochem Cell Biol. 2014;54:288-303.

6. Vienberg S, Geiger J, Madsen S, Dalgaard LT. MicroRNAs in metabolism. Acta Physiol (Oxf). 2017;219(2):346-61.

7. Sontakke SD, Mohammed BT, McNeilly AS, Donadeu FX. Characterization of microRNAs differentially expressed during bovine follicle development. Reproduction. 2014;148:271-83.

8. Farazi TA, Hoell Il, Morozov P, Tuschl T. MicroRNAs in human cancer. Adv Exp Med Biol. 2013;774:1-20.

9. Mitchell PS, Parkin RK, Kroh EM, Fritz BR, Wyman SK, Pogosova-Agadjanyan EL, Peterson A, Noteboom J, O'Briant KC, Allen A, et al. Circulating microRNAs as stable blood-based markers for cancer detection. Proc Natl Acad Sci U S A. 2008:105:10513-8.

10. He $Y$, Lin J, Kong D, Huang M, Xu C, Kim TK, Etheridge A, Luo Y, Ding Y, Wang K. Current state of circulating MicroRNAs as Cancer biomarkers. Clin Chem. 2015;61:1138-55.

11. Schwarzenbach H, Nishida N, Calin GA, Pantel K. Clinical relevance of circulating cell-free microRNAs in cancer. Nat Rev Clin Oncol. 2014;11:145-56.

12. Creemers EE, Tijsen AJ, Pinto YM. Circulating microRNAs: novel biomarkers and extracellular communicators in cardiovascular disease? Circ Res. 2012; 110:483-95.

13. Afonso MB, Rodrigues PM, Simao AL, Castro RE. Circulating microRNAs as potential biomarkers in non-alcoholic fatty liver disease and hepatocellular carcinoma. J Clin Med. 2016;5(3):30.

14. Guay C, Regazzi R. Circulating microRNAs as novel biomarkers for diabetes mellitus. Nat Rev Endocrinol. 2013;9:513-21.

15. Miura K, Miura S, Yamasaki K, Higashijima A, Kinoshita A, Yoshiura K, Masuzaki $\mathrm{H}$. Identification of pregnancy-associated microRNAs in maternal plasma. Clin Chem. 2010;56:1767-71.

16. Pritchard CC, Kroh E, Wood B, Arroyo JD, Dougherty KJ, Miyaji MM, Tait JF, Tewari M. Blood cell origin of circulating microRNAs: a cautionary note for cancer biomarker studies. Cancer Prev Res (Phila). 2012;5:492-7.

17. Leidner RS, Li L, Thompson CL. Dampening enthusiasm for circulating microRNA in breast cancer. PLoS One. 2013:8:e57841.

18. Haider BA, Baras AS, McCall MN, Hertel JA, Cornish TC, Halushka MK. A critical evaluation of microRNA biomarkers in non-neoplastic disease. PLoS One. 2014;9:e89565

19. Ioannidis J, Donadeu FX. Circulating miRNA signatures of early pregnancy in cattle. BMC Genomics. 2016;17:184

20. Ioannidis J, Donadeu FX. Circulating microRNA profiles during the bovine Oestrous cycle. PLoS One. 2016;11:e0158160.

21. Farrell D, Shaughnessy RG, Britton L, MacHugh DE, Markey B, Gordon SV. The identification of circulating MiRNA in bovine serum and their potential 
as novel biomarkers of early Mycobacterium avium subsp paratuberculosis infection. PLoS One. 2015;10:e0134310.

22. Hansen EP, Kringel $H$, Thamsborg SM, Jex A, Nejsum P. Profiling circulating miRNAs in serum from pigs infected with the porcine whipworm, Trichuris suis. Vet Parasitol. 2016;223:30-3.

23. Muroya S, Ogasawara H, Hojito M. Grazing affects Exosomal circulating MicroRNAs in cattle. PLoS One. 2015;10:e0136475.

24. Ahanda ML, Zerjal T, Dhorne-Pollet S, Rau A, Cooksey A, Giuffra E. Impact of the genetic background on the composition of the chicken plasma MiRNome in response to a stress. PLoS One. 2014:9:e114598.

25. Cheng HH, Yi HS, Kim Y, Kroh EM, Chien JW, Eaton KD, Goodman MT, Tait JF, Tewari M, Pritchard CC. Plasma processing conditions substantially influence circulating microRNA biomarker levels. PLoS One. 2013;8:e64795.

26. Ioannidis J, Donadeu FX. MicroRNA biomarkers of tissue function. GEO 2016. https://www.ncbi.nlm.nih.gov/geo/query/acc.cgi?acc=GSE84871.

27. Barturen G, Rueda A, Hamberg M, Alganza A, Lebron R, Kotsyfakis M, Shi BJ, Koppers-Lalic D, Hackenberg M. sRNAbench: profiling of small RNAs and its sequence variants in single or multi-species high-throughput experiments. Methods in Next Generation Sequencing. 2014; https:/doi.org/10.2478/mngs-2014-0001.

28. Barrett T, Wilhite SE, Ledoux P, Evangelista C, Kim IF, Tomashevsky M, Marshall KA, Phillippy KH, Sherman PM, Holko M, et al. NCBI GEO: archive for functional genomics data sets-update. Nucleic Acids Res. 2013;41:D991-5.

29. Elsik CG, Unni DR, Diesh CM, Tayal A, Emery ML, Nguyen HN, Hagen DE. Bovine genome database: new tools for gleaning function from the Bos taurus genome. Nucleic Acids Res. 2016;44:D834-9.

30. Griffiths-Jones S, Saini HK, van Dongen S, Enright AJ. miRBase: tools for microRNA genomics. Nucleic Acids Res. 2008;36:D154-8.

31. R Core Team. R: a language and environment for statistical computing. Vienna, Austria: R Foundation for Statistical Computing; 2013.

32. Robinson MD, McCarthy DJ, Smyth GK. edgeR: a Bioconductor package for differential expression analysis of digital gene expression data. Bioinformatics. 2010;26:139-40.

33. McCarthy DJ, Chen Y, Smyth GK. Differential expression analysis of multifactor RNA-Seq experiments with respect to biological variation. Nucleic Acids Res. 2012;40:4288-97.

34. Aken BL, Ayling S, Barrell D, Clarke L, Curwen V, Fairley S, Fernandez Banet J, Billis K, Garcia Giron C, Hourlier T, et al. The Ensembl gene annotation system. Database (Oxford). 2016; https://doi.org/10.1093/database/baw093.

35. Kozomara A, Griffiths-Jones S. miRBase: integrating microRNA annotation and deep-sequencing data. Nucleic Acids Res. 2011;39:D152-7.

36. Camacho C, Coulouris G, Avagyan V, Ma N, Papadopoulos J, Bealer K, Madden TL. BLAST+: architecture and applications. BMC Bioinf. 2009;10:421.

37. Landgraf P, Rusu M, Sheridan R, Sewer A, lovino N, Aravin A, Pfeffer S, Rice A, Kamphorst AO, Landthaler M, et al. A mammalian microRNA expression atlas based on small RNA library sequencing. Cell. 2007;129:1401-14.

38. Guo Z, Maki M, Ding R, Yang Y, Zhang B, Xiong L. Genome-wide survey of tissue-specific microRNA and transcription factor regulatory networks in 12 tissues. Sci Rep. 2014;4:5150.

39. Wang K, Yuan Y, Cho J-H, McClarty S, Baxter D, Galas DJ. Comparing the MicroRNA Spectrum between serum and plasma. PLoS One. 2012;7:e41561.

40. Hunter MP, Ismail N, Zhang X, Aguda BD, Lee EJ, Yu L, Xiao T, Schafer J, Lee ML, Schmittgen TD, et al. Detection of microRNA expression in human peripheral blood microvesicles. PLoS One. 2008;3:e3694.

41. Willeit P, Zampetaki A, Dudek K, Kaudewitz D, King A, Kirkby NS, CrosbyNwaobi R, Prokopi M, Drozdov I, Langley SR, et al. Circulating MicroRNAs as novel biomarkers for platelet activation. Circ Res. 2013;112:595-+.

42. Jin W, Dodson MV, Moore SS, Basarab JA, Guan LL. Characterization of microRNA expression in bovine adipose tissues: a potential regulatory mechanism of subcutaneous adipose tissue development. BMC Mol Biol. 2010;11:29.

43. Huang J, Ju Z, Li Q, Hou Q, Wang C, Li J, Li R, Wang L, Sun T, Hang S, et al. Solexa sequencing of novel and differentially expressed microRNAs in testicular and ovarian tissues in Holstein cattle. Int J Biol Sci. 2011;7:1016-26.

44. Kornfeld JW, Baitzel C, Konner AC, Nicholls HT, Vogt MC, Herrmanns K, Scheja L, Haumaitre C, Wolf AM, Knippschild U, et al. Obesity-induced overexpression of miR-802 impairs glucose metabolism through silencing of Hnf1b. Nature. 2013;494:111-5.

45. Higuchi C, Nakatsuka A, Eguchi J, Teshigawara S, Kanzaki M, Katayama A, Yamaguchi S, Takahashi N, Murakami K, Ogawa D, et al. Identification of circulating miR-101, miR-375 and miR-802 as biomarkers for type 2 diabetes. Metabolism. 2015;64:489-97.
46. Hao R, Su S, Wan Y, Shen F, Niu B, Coslo DM, Albert I, Han X, Omiecinski CJ. Bioinformatic analysis of microRNA networks following the activation of the constitutive androstane receptor (CAR) in mouse liver. Biochim Biophys Acta. 1859;2016:1228-37.

47. Church RJ, Otieno M, McDuffie JE, Singh B, Sonee M, Hall L, Watkins PB, Ellinger-Ziegelbauer $\mathrm{H}$, Harrill AH. Beyond miR-122: identification of MicroRNA alterations in blood during a time course of hepatobiliary injury and biliary hyperplasia in rats. Toxicol Sci. 2016;150:3-14.

48. Wolenski FS, Shah P, Sano T, Shinozawa T, Bernard H, Gallacher MJ, Wyllie SD, Varrone G, Cicia LA, Carsillo ME, et al. Identification of microRNA biomarker candidates in urine and plasma from rats with kidney or liver damage. J Appl Toxicol. 2016;37(3):278-86.

49. Fatima A, Lynn DJ, O'Boyle P, Seoighe C, Morris D. The miRNAome of the postpartum dairy cow liver in negative energy balance. BMC Genomics. 2014;15:279.

\section{Ready to submit your research? Choose BMC and benefit from:}

- fast, convenient online submission

- thorough peer review by experienced researchers in your field

- rapid publication on acceptance

- support for research data, including large and complex data types

- gold Open Access which fosters wider collaboration and increased citations

- maximum visibility for your research: over $100 \mathrm{M}$ website views per year

At BMC, research is always in progress.

Learn more biomedcentral.com/submissions 